\title{
Solar PV nowcasting based on skycamera observations
}

\author{
Oleksandra Voronych ${ }^{1}$, Robert Höller ${ }^{1}$, Germanno Longhi Beck ${ }^{1}$, and Wolfgang Traunmüller ${ }^{2}$ \\ ${ }^{1}$ University of Applied Sciences Upper Austria, Department of Sustainable \\ Energy Systems, Wels, 4600, Austria \\ ${ }^{2}$ BLUE SKY Wetteranalysen, Attnang-Puchheim, 4800, Austria \\ Correspondence: Robert Höller (robert.hoeller@fh-wels.at)
}

Received: 12 January 2019 - Accepted: 20 February 2019 - Published: 28 February 2019

\begin{abstract}
In recent years the installation of PV systems has increased dramatically in many countries. Annual global installed power has already reached more than $400 \mathrm{GW}$ in 2017. A major challenge for operators is that PV system output is highly fluctuating due to cloud movements and other atmospheric influences. Forecasting of solar irradiation and PV power on different time scales will, therefore, become more and more important for different users. As part of the Austrian PV-go-Smart project, several skycameras have been installed in the region of Upper Austria. In this paper we show differences and advantages of two skycams and their image qualities. Algorithms for the detection of clouds, cloud movement, and GHI forecasting have been developed and validated with ground observation at the Wels site. This work focusses on image quality issues related to short-term irradiance forecasting using all-sky cameras, in particular the influence on raindrops on forecast performance.
\end{abstract}

\section{Introduction}

As the penetration of renewable energy sources in the power system grows, there is an emerging need for accurate forecasting of solar irradiation. Forecasting methods varying depending on their application, timescales (i.e. short-term or long-term forecasts), and spatial coverage. Short-term forecasts are usually based on remote sensing data or satellite data, while long-term forecasts are based on statistical and numerical weather prediction (NWP) methods. All-sky cameras provide a visual measurement of the dome of the entire sky, and it can capture clouds and sudden changes in the motion of clouds distributed over an area of $1-15 \mathrm{~km}$, depending on cloud height. The cameras used in this research are the SRF-02 produced by EKO Instruments and the Vivotek FE9391-EV. The cameras are installed on rooftops in Wels, Austria, with a distance of $2.5 \mathrm{~km}$ between the two installations. The appearance of the raindrops on the camera dome and consequently on the image can gradually influence forecast performance.

In general, the all-sky camera system makes use of the information from the visible sky and can predict cloud events or absence of clouds. The forecasting error is increasing with increased time horizon. Important to notice is that even though the forecasting error for very short lead times is smaller than for longer lead times, the forecast begins to outperform the persistence method only with a lead time of 5 min and higher. The key tasks of a short-term forecasting system are cloud detection and cloud motion estimation. For the cloud detection, a fundamental difference in light scattering by clouds in comparison to the clear sky applies. The approach based on the simple red-blue ratio and red-blue ratio corrected with clear sky image red-blue ratio were found to be the most fitting to detect the clouds in various weather and illumination situations (Shields et al., 1993). Among a variety of cloud motion estimation techniques that were developed in recent years, the cross-correlation (Chow et al., 2011) was found to be the most suitable for the scope of the work. Although short-term forecasting with all-sky cameras shows good and promising results, there is a list of issues: presence of raindrops on the camera's glass dome, cloud enhancement effect, variability in clouds optical properties, cloud spontaneous formation and dissolution, an influence of different cloud types on the forecast performance, multiple cloud layers. 

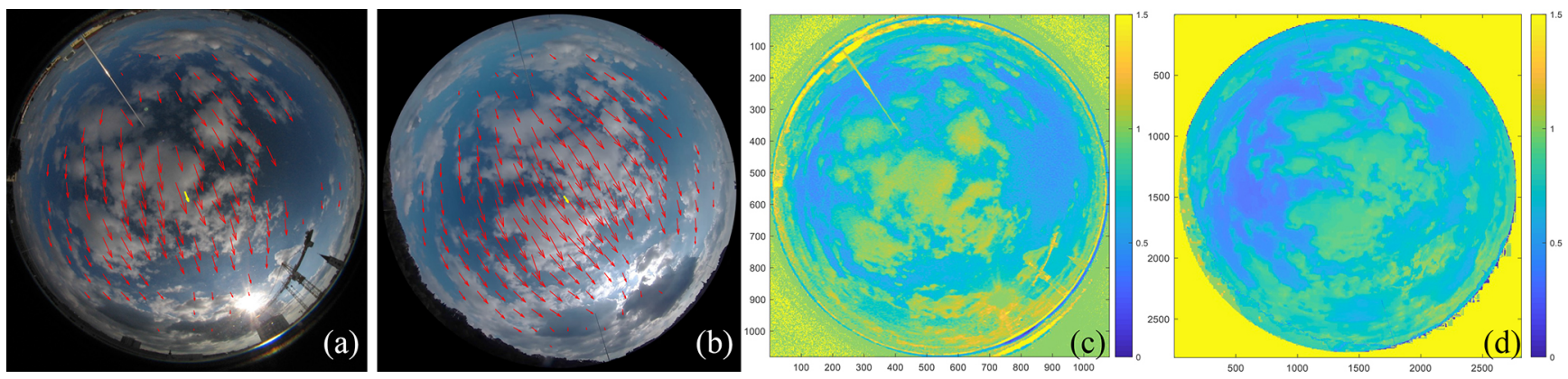

Figure 1. Cloud motion estimation (a, b) and cloud detection images (c, d) for the EKO (a, c) and the Vivotek (b, d).

(a)

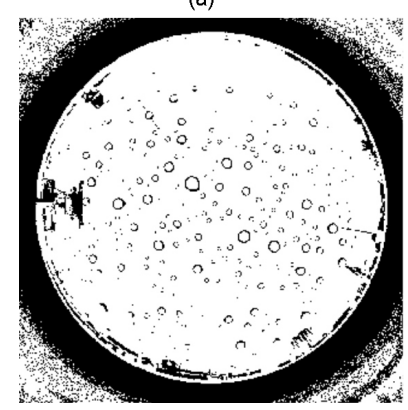

(b)

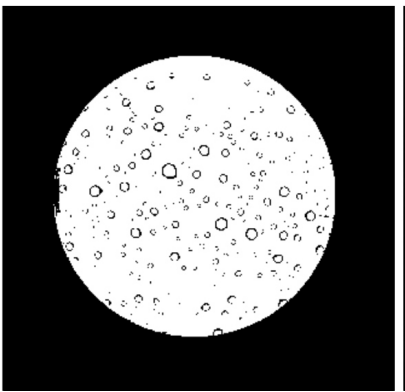

(c)

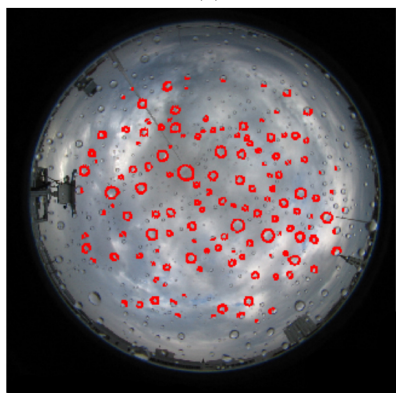

Figure 2. Raindrop detection. From (a) to (c): binary image, clipped binary image, final raindrop decision image.

\section{Comparison of two different cameras}

Within the scope of this work is also to apply a low-cost and reliable camera system. Here, the performance of the EKO SRF-02 camera was compared with the performance of the commercial surveillance camera Vivotek FE9391-EV. Both cameras are equipped with fisheye lenses and are capable of taking photos of the entire sky-dome. The images from both cameras were compared through the two key tasks in shortterm solar irradiance forecasting: cloud detection and cloud motion estimation (Fig. 1). Cloud detection functions better with images from the EKO camera, mainly due to the higher span of the red-blue ratio values. Both cameras showed similar performance for cloud motion estimation and both cameras provide good quality all-sky images for solar-irradiance forecasting.

\section{Raindrop detection}

Raindrops are intervening with the cloud decision algorithm as well as with cloud motion estimation. For this reason, reliable quality control of the images is required. Hence, it is of great importance to develop a reliable raindrop detection method for the all-sky images. There is one method for raindrop detection present in the literature (Kazantzidis et al., 2012). The method uses the fact that the raindrops standing on the perimeter of the hemispheric dome distort the circularity of the shape of the dome in the image. This method can provide a quick quality check, but it is not suitable for all conditions (e.g. most of the raindrops are not close to the dome perimeter) and all installation setups (e.g. when there are many horizon objects with different shape and height present in the image), and it has a low sensitivity.

The method used in this work is based on detecting dark (almost black) raindrop edges that have the pixel values very close to zero on the entire image. This allows detecting raindrop edges after the image is converted with a very low threshold value to a binary image (Fig. 2). Afterward, all remaining objects are tracked using the Moore-Neighbor tracing algorithm modified by Jacob's stopping criteria (Gonzales et al., 2004). After all elements are identified, based on object properties (a raindrop edge is a crescent-shaped object with certain size) the decision is made whether the object is a raindrop edge or not. All very small elements are treated as image noise.

Finally, the output of the method is a number of detected raindrop edges, which is normally from 1.5 to 2 times higher than the number of raindrops on the image. It is important to mention that concerning the short-term irradiance forecasting the exact number of raindrops detected on the image is less important than the absolute area on the image covered by them. Each water droplet on the camera dome acts as a spherical lens. It reflects light from a large field of view towards the camera. Hence, despite raindrops are transparent, they distort and reflect the background clouds to that extent 


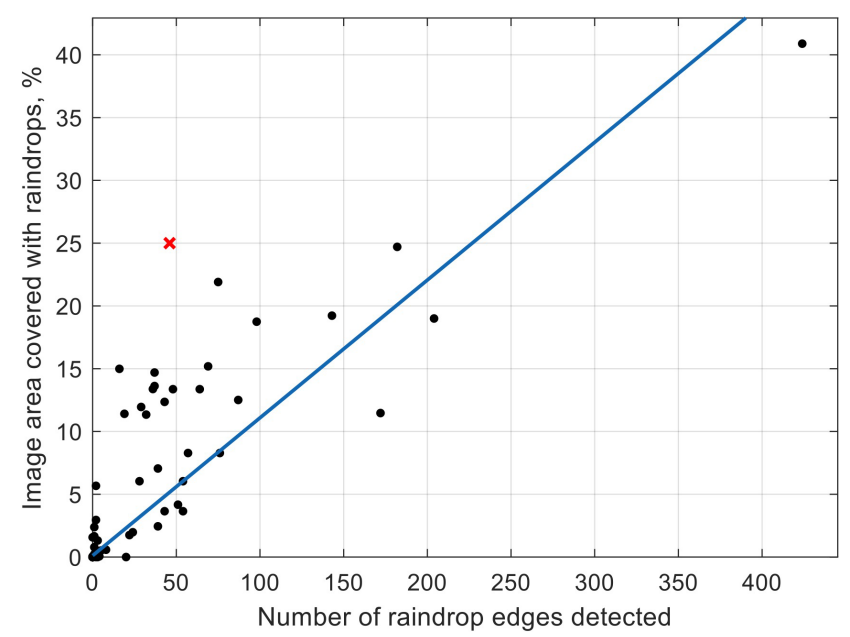

Figure 3. Correlation between the number of raindrop edges detected by the algorithm and the total area covered by raindrops.

that these parts of the image cannot be further used for the forecast applications.

In order to check the algorithm performance, a batch of images with different background weather and illumination conditions was selected among all available data. The algorithm was able to detect most of the raindrops of various shapes and sizes and for all tested circumstances. However, some minor raindrops remain undetected. This implies to very small drops (with the diameter on image less than 10 pixels) and water drops with a pale edge, hardly noticeable even when examining the image with the naked eye. Also, in minor cases small very dark parts of cloud edges were misinterpreted by the algorithm as raindrops.

During the verification of the performance of the method, a correlation between the number of raindrop edges detected and the total area covered by raindrops was established (see Fig. 3). This correlation can be further used for the assessment of the image quality in the forecast applications.

After heavy rain, when the complete image is covered with raindrops, the total area of all raindrops on the photo is close to $10 \%-25 \%$ of the total area of the visible sky area on the image. With such a ratio of raindrop coverage of the dome it is impossible to produce a reliable short-term solar irradiance forecast. Even the raindrop coverage of $5 \%$ introduces a significant error in forecast results. Hence, it is very important with the result of the forecast algorithm to add a notification on the quality of the images taken for generating each result.

\section{Conclusions}

This work is dedicated to the image quality issues related to short-term irradiance forecasting using all-sky cameras. Two different cameras were compared, and it was shown that both expansive all-sky camera and budget surveillance camera have shown good performance, and both can be used for short-term solar irradiance forecasting. It was shown that with a raindrop coverage of the dome of $5 \%-10 \%$, it is impossible to produce a reliable short-term solar irradiance forecast. Therefore, a method for raindrop detection was developed and validated. Overall, the method is performing well and able to detect a large part of raindrops in the image with an insignificant share of misclassified elements, and it is recommended to be implemented in short-term solar irradiance forecasting algorithm as a part of image quality assessment.

Data availability. Data are currently not publicly available.

Author contributions. RH supervised this research and OV developed the model code and performed the simulations. GLB developed the hardware and WT supported the measurements and provided resources. OV and $\mathrm{RH}$ prepared the manuscript.

Competing interests. The authors declare that they have no conflict of interest.

Special issue statement. This article is part of the special issue "18th EMS Annual Meeting: European Conference for Applied Meteorology and Climatology 2018". It is a result of the EMS Annual Meeting: European Conference for Applied Meteorology and Climatology 2018, Budapest, Hungary, 3-7 September 2018.

Acknowledgements. This work is part of the project PV-goSmart and was funded in the framework of "Digitalisierung" of Strategie Innovatives Oberösterreich 2020.

Edited by: Sven-Erik Gryning

Reviewed by: Alexandros Charalambides and one anonymous referee

\section{References}

Chow, C. W., Urquhart, B., Lave, M., Dominguez, M., Kleissl, J., Shields, J., and Washom, B.: Intra-hour forecasting with a total sky imager at the UC San Diego solar energy testbed, Solar Energy, 85, 2881-2893, https://doi.org/10.1016/j.solener.2011.08.025, 2011.

Gonzales, R. C., Woods, R. E., and Eddins, S. L.: Digital Image Processing Using MATLAB, New Jersey, Pearson Prentice Hall, 2004

Kazantzidis, A., Tzoumanikas, P., Bais, A. F., Fotopoulos, S., and Economou, G.: Cloud detection and classification with the use of whole-sky ground-based images, Atmos. Res., 113, 80-88, https://doi.org/10.1016/j.atmosres.2012.05.005, 2012. 
Shields, J. E., Johnson, R. W., and Koehler, T. L.: Automated Whole Sky Imaging Systems for Cloud Field Assessment, 4th Symp. on Glob. Chang. Stud., 17-22 January 1993, Anaheim, California, American Meteorological Society, 1993. 